\title{
Nuclear Emulsion Films with HIMAC heavy ion beams
}

\author{
Moegi Okuyama ${ }^{a}{ }^{*}$, Atsushi lyono ${ }^{b}$, Saya Yamamoto $a$, Koshiro Izumi $a$, Shigeki \\ Aoki $^{c}$, Satoshi Kodairad
}

a Graduate school of science, Okayama University of Science, 700-005, 1-1 Ricai-cho, Kita-ku, Okayama, Japan

b Faculty of Science, Okayama University of Science, 700-005, 1-1 Ricai-cho, Kita-ku, Okayama, Japan

cFaculty of Human Development, Kobe University, 657-8501, 3-11 Tsurukabuto, Nada-ku, Kobe, Japan

d National Institute of Radiological Sciences

263-8885, 4-9-1, Anagawa, Inage-ku, Chiba-shi, Chiba, Japan

E-mail: s20sm02omeous.jp

By adding suitable amount of Rhodium compound $\left(\mathrm{Na}_{3} \mathrm{RhCl}_{6} \cdot 5 \mathrm{H}_{2} \mathrm{O}\right)$ during the production of nuclear emulsion gel, it enabled to reduce the sensitivity of the nuclear emulsion films and realized the selection of heavy nuclei by using image processing system suitable for minimum ionized particles. We have carried out the beam exposure to desensitized nuclear emulsion films in October, 2019 at Heavy Ion Medical Accelerator in Chiba(HIMAC). When charged particles passed through the nuclear emulsion films, the tracks were measured as a series of silver grains of which size is typically less than one micrometer, and we traditionally determine their charge amount by measuring ionization loss signals such as grain density, deltaray count. In this study, we measured the energy losses of heavy ion beams in desensitized nuclear emulsion films exposed horizontally to emulsion layer, and we have estimated the desensitization effect for heavy ion detections.

\section{*Presenter}




\section{Introduction}

A nuclear emulsion is a highly sensitized type of photographic film for observing high-speed charged particles such as cosmic rays and elementary particles. On a both side of a plastic film (180 $\mu \mathrm{m}$ thickness), an nuclear emulsion gel made by mixing silver bromide crystals with gelatin is applied so that the thickness when dried is about $70 \mu \mathrm{m}$ per each side. When the charged particles pass through the nuclear emulsion film, the silver bromide in the emulsion is exposed to electrons, leaving a series of black spot-like particles (grains) in the nuclear emulsion. After that, the development process leaves observable tracks with an optical microscope with a spatial resolution of $1 \mu \mathrm{m}$. By this method, it is possible to identify the nuclear species and measure the mass and energy from the incident charged particles.

The nuclear emulsion is a highly sensitive track detector that is sensitive to particles with the minimum ionization loss, and has been used as a track detector in elementary particles, nuclei, and space experiments. On the other hand, because of its high sensitivity, it also has the property of making image processing difficult due to the $\delta$ rays generated along the tracks when detecting heavy particles by using high speed image processing system.

In order to control the sensitivity of the nuclear emulsion and determine the sensitivity conditions of the nuclear emulsion suitable for detecting heavy particles in space, beam irradiation experiments were conducted in October 2019 at the accelerator HIMAC of the National Institute of Radiological Sciences.

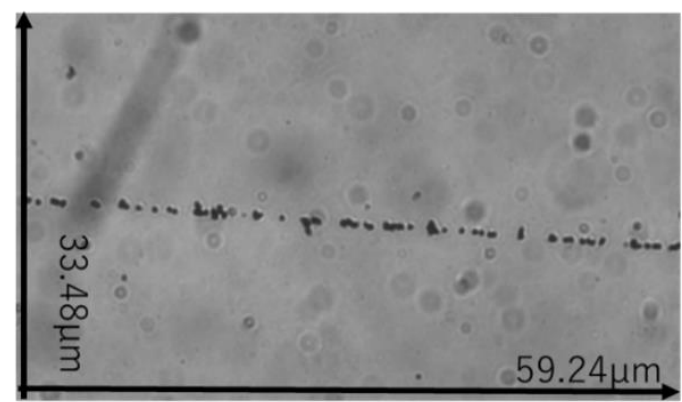

Fig1.C beam

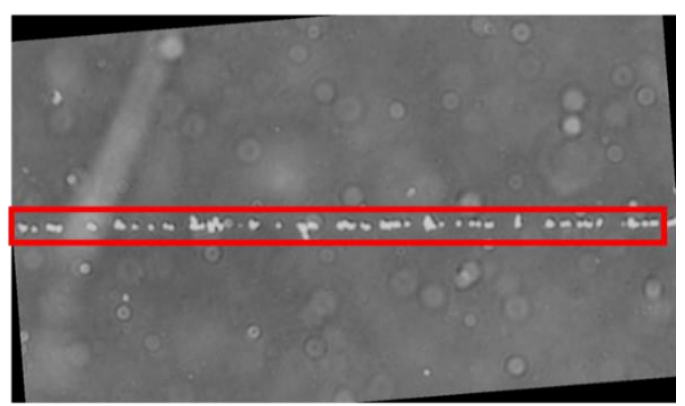

Fig2. Image rotation, inverted $\mathrm{C}$ beam

\section{Method}

$5 \mathrm{~cm} \times 5 \mathrm{~cm}$ size nuclear emulsion films ( $70 \mu \mathrm{m}$ nuclear emulsion applied to both sides of a $180 \mu \mathrm{m}$ acrylic plate) whose sensitivity was adjusted by controlling the amount of chemical substances and gelatin, were irradiated with heavy particle beams using HIMAC facility. The carbon and iron beams were horizontally exposed, whose energies are $400 \mathrm{MeV} / \mathrm{u}$ and $500 \mathrm{MeV}$ /u, respectively.

By adding the $\mathrm{Rh}$ compound $\left(\mathrm{Na}_{3} \mathrm{RhCl}_{6} \cdot 5 \mathrm{H}_{2} \mathrm{O}\right)$ during the production of the nuclear emulsion, the sensitivity of the nuclear emulsion can be controlled to be lower than ordinal nuclear emulsion gel. Since the Rh compound is considered to have a property of inhibiting the movement of electrons by the sulfur compound during the formation of photosensitive latent image nucleation, the sensitivity can be lowered as the amount of addition is larger. In this study, observations were made on a nuclear emulsion prepared by adding $5.0 \mu \mathrm{mol} / \mathrm{Ag}$ mol of the $\mathrm{Rh}$ compound.

The tracks (grains) remaining in the nuclear emulsion films were observed with an optical microscope (objective 100x lens), and the nuclear emulsion was divided into the top, middle, and bottom part from the beam upstream when acquiring images. The number of grains in the image was measured, the number of grains per $100 \mu \mathrm{m}$ was obtained, and the amount of ionization loss 
of each heavy particle was determined in each part to distinguish their differences of energy losses at each part.

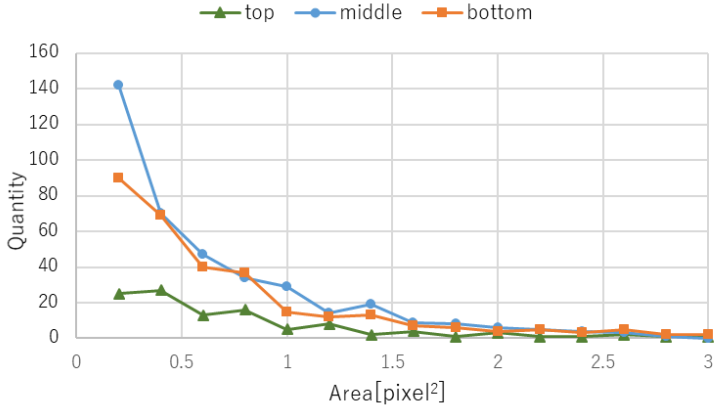

Fig3. Area distribution of $\mathrm{C}$ beam

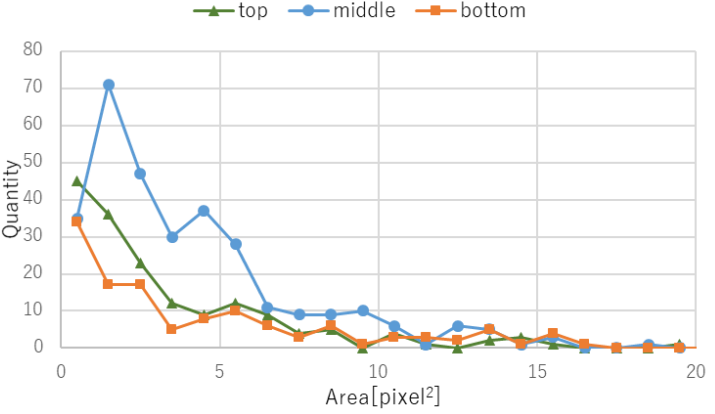

Fig4. Area distribution of Fe beam

At this time, as a new approach, the area of each grain was determined after performing image rotation, black-and-white inversion, and threshold processing on the image of each grain recorded in the image. Then, instead of the conventional method of counting the number of grains, the total area is obtained, standardized by the referenced area, and defined as the amount of ionization losses. Fig. 3 and 4 show the distribution of grain areas in the top, middle, and bottom regions for $\mathrm{Fe}$ and $\mathrm{C}$ beams, respectively. It can be seen that the tracks are composed not only of a single grain but also of grains of various sizes. In particular, the Fe beam in Fig. 4 shows a tendency that a population of grains is more predominant than a single grain.

\section{Resluts}

Fig. 5 shows the measurement results for the $\mathrm{C}$ and $\mathrm{Fe}$ beams at three locations top, middle, and bottom of the nuclear emulsion having the grain density determined by the above method. The results are shown after converting to the conventional method of silver particle density (grain / $100 \mu \mathrm{m}$ ). As a result, in both of the beams, it is possible to capture the ionized amount of change in the

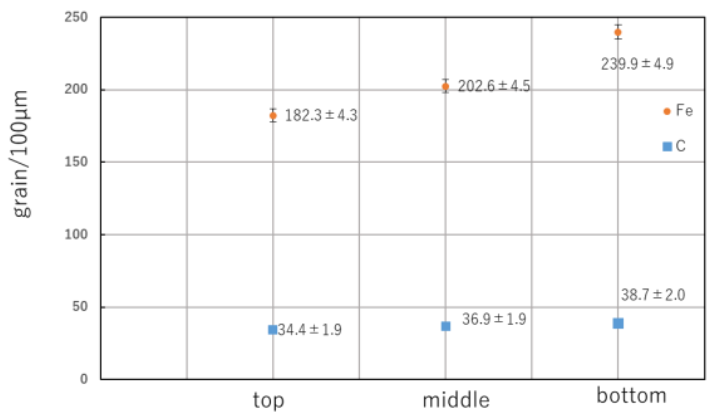

Fig5. C, Fe beam grain density $5 \mathrm{~cm}$ nuclear emulsion of suppressing the influence of $\delta$ line due to low sensitivity, are obtained grain counting or equivalent accuracy. According to the theoretical formula of ionization loss, it is $52.63 \mathrm{MeV} / \mathrm{u}$ for a C beam of $400 \mathrm{MeV} / \mathrm{u} .37 .43 \mathrm{MeV} / \mathrm{u}$ for a $500 \mathrm{MeV} / \mathrm{u}$ Fe beam. Energy is lost, I think of could be observed.

\section{Conclusions}

Desensitized nulcear emalsion films were exposed to HIMAC heavy ion beams and their sensitivities were also exmined by measuring ionization losses in the three part of exposed emulsion film area. The desensitization of nuclear emulsion film could open the study of cosmic ray nuclei by using high speed image processing with high statistics.

We developed new grain count method by integration of grain or grain-clump area and deviding them by standard grain area. It well operated in the analysis of horizontal beams. 


\section{Acknowledgements}

This work was carried out by the joint usage / research program of the Institute of Materials and Systems for Sustainability(IMaSS), Nagoya University

\section{References}

[1] S. Yamamoto et al., Bull. Soc. Photogr. Imag. Japan. (2019) Vol. 29 No. 2: 20-26

[2] Saya Yamamoto, (2020). Study of cosmic gamma ray detection and cosmic nuclei identification by balloon-borne experiment with nuclear emulsion film and desensitized nuclear emulsion film (Publication No. 35302 Demandant's Exhibit No. Science 115) [Doctoral dissertation, Okayama University of Science].AROUS

[3] Minoru Oda, Uchusen, (Shokabo, 1960), pp.108-115

[4] Isao Miura, Koichi Suga, Tsuneo Matano,Housyasenkeisokugaku, (Shokabo, 1960), pp.177-200

[5] William R. Leo, Techniques for Nuclear and Particle Physics Experiments, pp.25-27 